\title{
Hyponatremia and polyuria in an older woman
}

\section{Kevin Afra MD, Matthew T. James MD PhD}

A 77-year-old woman was admitted to hospital following 2 weeks of malaise, vomiting and decreased oral intake. She had multiple comorbidities, including coronary artery disease, cerebrovascular disease, dyslipidemia and long-standing hypertension, for which she was taking a $\beta$-blocker and a nondihydropyridine calcium-channel blocker. She also had a history of adrenal insufficiency treated with longterm prednisone and hypothyroidism treated with levothyroxine. She had been admitted to hospital 5 times over the preceding 2 years because of hyponatremia. These episodes had been attributed to hypovolemia and relative adrenal insufficiency and had been treated with intravenous fluids and stress doses of steroids. Complicating her story was a series of surgical procedures for chronic sinusitis. Kidney disease had been documented, with a baseline serum creatinine of $100 \mu \mathrm{mol} / \mathrm{L}$ (estimated glomerular filtration rate $47 \mathrm{~mL} / \mathrm{min}$ per $1.73 \mathrm{~m}^{2}$ ), and ultrasonography had previously shown renal asymmetry $(6.7 \mathrm{~cm}$ v. $9.3 \mathrm{~cm}$ on the right); these findings, together with her history of atherosclerotic events, had suggested renovascular disease. Magnetic resonance angiography performed before the current admission had shown critical stenosis of her left renal artery.

On the current presentation, the patient had hypertension (blood pressure 158/107 $\mathrm{mm} \mathrm{Hg}$ ) but was hypovolemic, with dry mucous membranes and a low jugular venous pressure. Findings on cardiac, respiratory and abdominal examinations were otherwise unremarkable. Laboratory findings showed hyponatremia (serum sodium 114 [normal 133-145] mmol/L), hypokalemia (serum potassium 2.9 [normal 3.35.1] $\mathrm{mmol} / \mathrm{L}$ ) and an elevated serum creatinine level (135 [normal 35-100] $\mu \mathrm{mol} / \mathrm{L})$.

We started intravenous treatment with normal saline, potassium and corticosteroids. The patient's sodium level returned to normal over the next 4 days. Results of endocrine tests showed a low morning cortisol level (136 [normal > 500] $\mathrm{nmol} / \mathrm{L}$ ), a normal thyroid-stimulating hormone level (0.4 [normal 0.2-6.0] mU/L), but a low level of free thyroxine (9 [normal 10-25] pmol/L). Her cortisone and levothyroxine doses were increased. However, when we subsequently stopped intravenous fluids and potassium supplementation, the patient had a recurrence of hyponatremia, hypokalemia and prerenal azotemia. During this time, she continued to experience polyuria (urine output 3-5 L/d) and hypertension (systolic pressure 150-165 $\mathrm{mm} \mathrm{Hg}$ and diastolic pressure 80$105 \mathrm{~mm} \mathrm{Hg}$ ).

\section{What is your diagnosis?}

a. Central diabetes insipidus due to trauma

b. Nephrogenic diabetes insipidus resulting from hypokalemia

c. Panhypopituitarism as a consequence of sinus surgery

d. Hyponatremic-hypertensive syndrome secondary to unilateral stenosis of the renal artery

The diagnosis in this case is (d) hyponatremichypertensive syndrome secondary to unilateral stenosis of the renal artery. To reach the diagnosis, we first evaluated the cause of the hyponatremia (Figure 1). The patient's measured serum osmolality was 248 (normal 280-300) $\mathrm{mmol} / \mathrm{kg}$. Her calculated serum osmolality - calculated as 2 (serum sodium) + serum glucose + urea - was $2(114)+5+5=238 \mathrm{mmol} / \mathrm{kg}$. A low serum osmolality indicated hypo-osmolar hyponatremia, as opposed to iso-osmolar or hyperosmolar hyponatremia. Neither central nor nephrogenic diabetes insipidus would be consistent with hypoosmolar hyponatremia.

Hyponatremia is the result of excessive free water in relation to body sodium. Although our patient presented with mild acute kidney injury on chronic kidney disease, the degree of renal impairment was not sufficient to impair excretion of free water by the kidneys. Some causes of hyponatremia, namely primary polydipsia or low solute intake, are associated with low levels of antidiuretic hormone and appropriately dilute urine. Minimum urine osmolality in healthy individuals
Competing interests: Matthew James received speaker fees from Amgen for a presentation on acute kidney injury at a nephrology conference. No competing interests declared by Kevin Afra.

This article has been peer reviewed.

Correspondence to: Matthew T. James, mjames@ucalgary.ca

CMAJ 2013. DOI:10.1503 /cmaj.121757 
is about $50 \mathrm{mmol} / \mathrm{kg}$. In our patient, the urine osmolality was 178 (normal 50-1200) mmol/kg, which indicated antidiuretic-hormone activity. The patient's urine sodium was $41 \mathrm{mmol} / \mathrm{L}$ and urine potassium $20 \mathrm{mmol} / \mathrm{L}$ (normal levels are diet dependent).

We next sought to determine whether there was a physiologic stimulus for secretion of antidiuretic hormone, such as hypovolemia, or a low effective circulating volume state. A low urine sodium concentration, generally less than $20 \mathrm{mmol} / \mathrm{L}$, tends to accompany such conditions; however, our patient's urine sodium level was elevated. Although elevated levels may be seen in SIADH (syndrome of inappropriate antidiuretic hormone secretion), adrenal insufficiency and hypothyroidism, they may also be seen in the setting of hypovolemia when renal sodium losses are present. The recurrence of hyponatremia and volume depletion in the setting of cortisone and levothyroxine therapy pointed to another cause contributing to our patient's clinical presentation.

The features of hypovolemia with concomitant polyuria and elevated urine sodium levels suggested renal losses. The patient had no his-

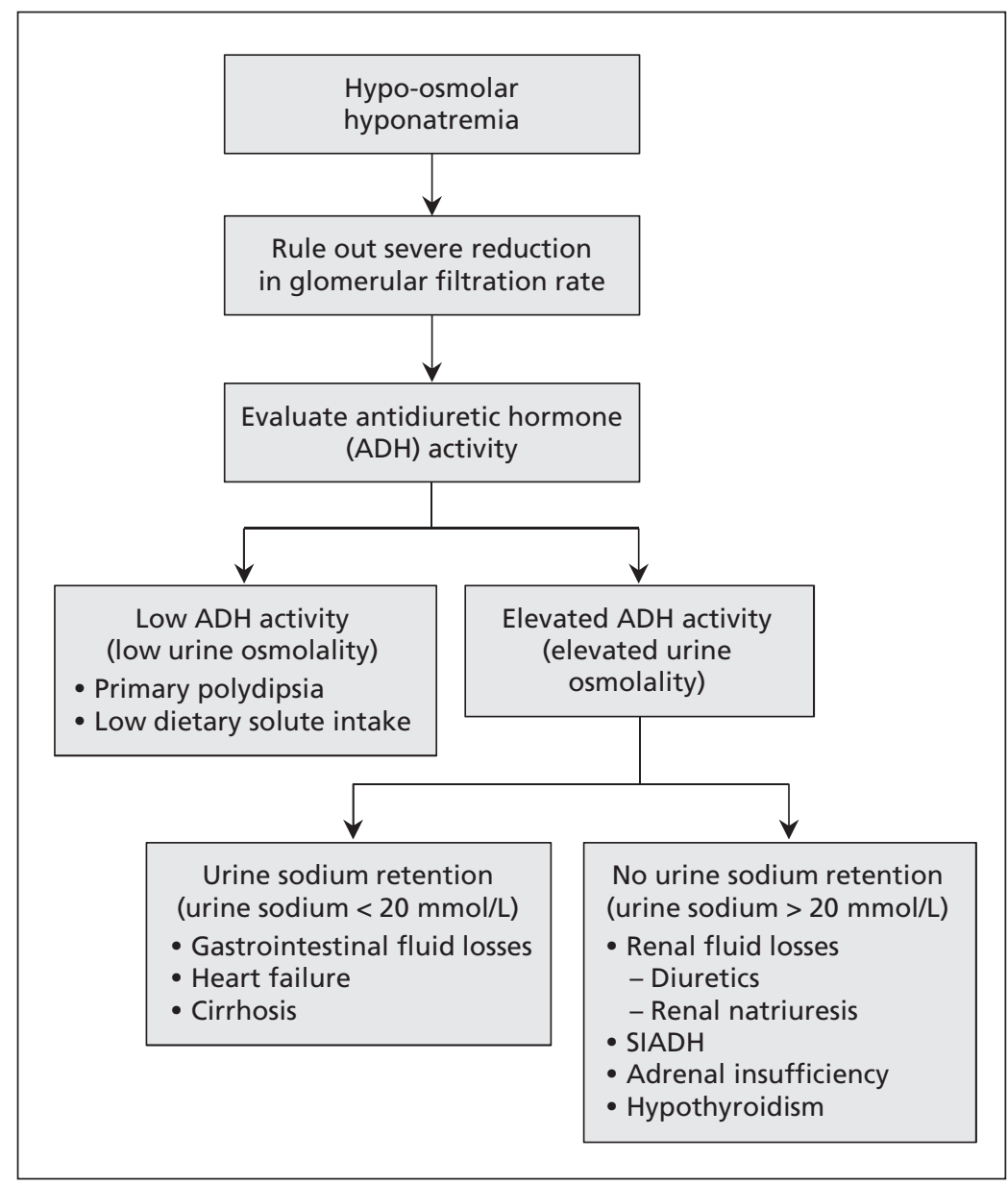

Figure 1: Physiologic approach to hypo-osmolar hyponatremia. SIADH = syndrome of inappropriate antidiuretic hormone secretion. Source: Wakil et al. ${ }^{1}$ tory of diuretic use to suggest drug-induced renal losses, which left renal natriuresis as the most likely problem. Taking into account her hypertension and critical stenosis of the left renal artery, we diagnosed hyponatremic-hypertensive syndrome.

\section{Which study confirms the diagnosis?}
a. Urine excretion in a 24-hour collection
b. Elevated renin activity
c. Water deprivation study
d. Cosyntropic stimulation test

Hyponatremic-hypertensive syndrome is associated with elevated renin activity generated by the ischemic kidney (b). Our patient's renin activity was markedly elevated (2.96 [normal 0.08-0.64] $\mathrm{ng} / \mathrm{L}$ per second), which was consistent with the diagnosis of hyponatremic-hypertensive syndrome. A water deprivation study would be useful to confirm diabetes insipidus if the patient had polyuria and hypernatremia. Measurement of urine excretion in a 24-hour collection would only confirm the renal sodium losses already revealed by her polyuria and spot urine sodium concentrations. Although a cosyntropin stimulation test and thyroid-stimulating hormone level may have some diagnostic utility to rule out adrenal insufficiency and hypothyroidism, the recurrence of hyponatremia despite cortisone and levothyroxine replacement therapy reduced the likelihood that these tests would help us understand the problem.

\section{Discussion}

\section{Diagnosis and epidemiology}

Patients with hyponatremic-hypertensive syndrome may present with headache, polyuria, polydipsia, weight loss or seizures. These symptoms are accompanied by hypertension that is often severe (mean 228/124 mm Hg in one case series) and associated with a postural drop. ${ }^{2}$ Hyponatremia, hypokalemia, and elevated urine osmolality and urine sodium levels are in keeping with the diagnosis (Figure 1). The combination of hypertension, polyuria and hyponatremia, in conjunction with asymmetric renal artery disease and elevated renin activity, confirms the diagnosis of hyponatremic-hypertensive syndrome. The population-based incidence of the syndrome is unknown; however, one case series identified 32 people who met criteria for the diagnosis from a source population of 350000 followed for 17 years. ${ }^{2,3}$ 


\section{Pathophysiology}

The pathophysiology of the syndrome involves the interplay between unilateral renal ischemia, the renin-angiotensin-aldosterone system and responses in the contralateral kidney (Figure 2). In the setting of severe unilateral renal hypoperfusion, whether from critical stenosis of a single renal artery or asymmetric bilateral stenosis, the ischemic kidney generates high levels of renin, which leads to elevated plasma angiotensin II levels. Systemic blood pressure rises as a result of the angiotensin II activity, a physiologic attempt to restore perfusion to the ischemic kidney. The unaffected contralateral kidney is exposed to a much higher perfusion pressure than it requires, which leads to pressure natriuresis in an attempt to autoregulate. The pressure natriuresis leads to renal sodium wasting and the clinical hypovolemia typically seen in patients with hyponatremic-hypertensive syndrome. Volume depletion further stimulates renin activity, which contributes to a feedback loop. ${ }^{4}$

Angiotensin II also stimulates aldosterone secretion from the adrenal glands, which leads to renal potassium excretion and resultant hypokalemia. ${ }^{4}$ Volume contraction and elevated angiotensin II levels stimulate the release of antidiuretic hormone, which results in thirst and polydipsia. ${ }^{2,4}$ The antidiuretic hormone activity, along with excess free water intake from polydipsia relative to urine sodium wasting, results in hyponatremia.

\section{Management}

In a case report by Atkinson and colleagues, ${ }^{4}$ blockade of the renin-angiotensin-aldosterone system with the angiotensin-converting-enzyme (ACE) inhibitor captopril was effective in treating hyponatremic-hypertensive syndrome in a 52-year-old woman with unilateral occlusion of the renal artery. The woman underwent unilateral nephrectomy with no recurrence of the syndrome and no need for ongoing pharmacotherapy. Definitive therapy through surgical or percutaneous revascularization, or removal of the ischemic kidney has been described in most case reports. In the largest case series, involving 32 patients, ${ }^{2}$ many were given antihypertensive therapy alone, although the long-term effectiveness of this approach was not reported.

The new class of antidiuretic hormone receptor antagonists (vaptan drugs) have been shown to raise serum sodium concentrations in euvolemic and hypervolemic causes of hyponatremia, including SIADH, heart failure and cirrhosis. However, antagonism of antidiuretic hormone may be harmful in hypovolemic states, and vaptan drugs are thus contraindicated in hypovolemic conditions such as hyponatremic-hypertensive syndrome. ${ }^{5}$

In our patient, medical therapy was started with sodium chloride tablets ( $2 \mathrm{~g} / \mathrm{d}$ orally) to replace renal sodium losses and help maintain euvolemia. Blockade of the renin-angiotensinaldosterone system with an ACE inhibitor was

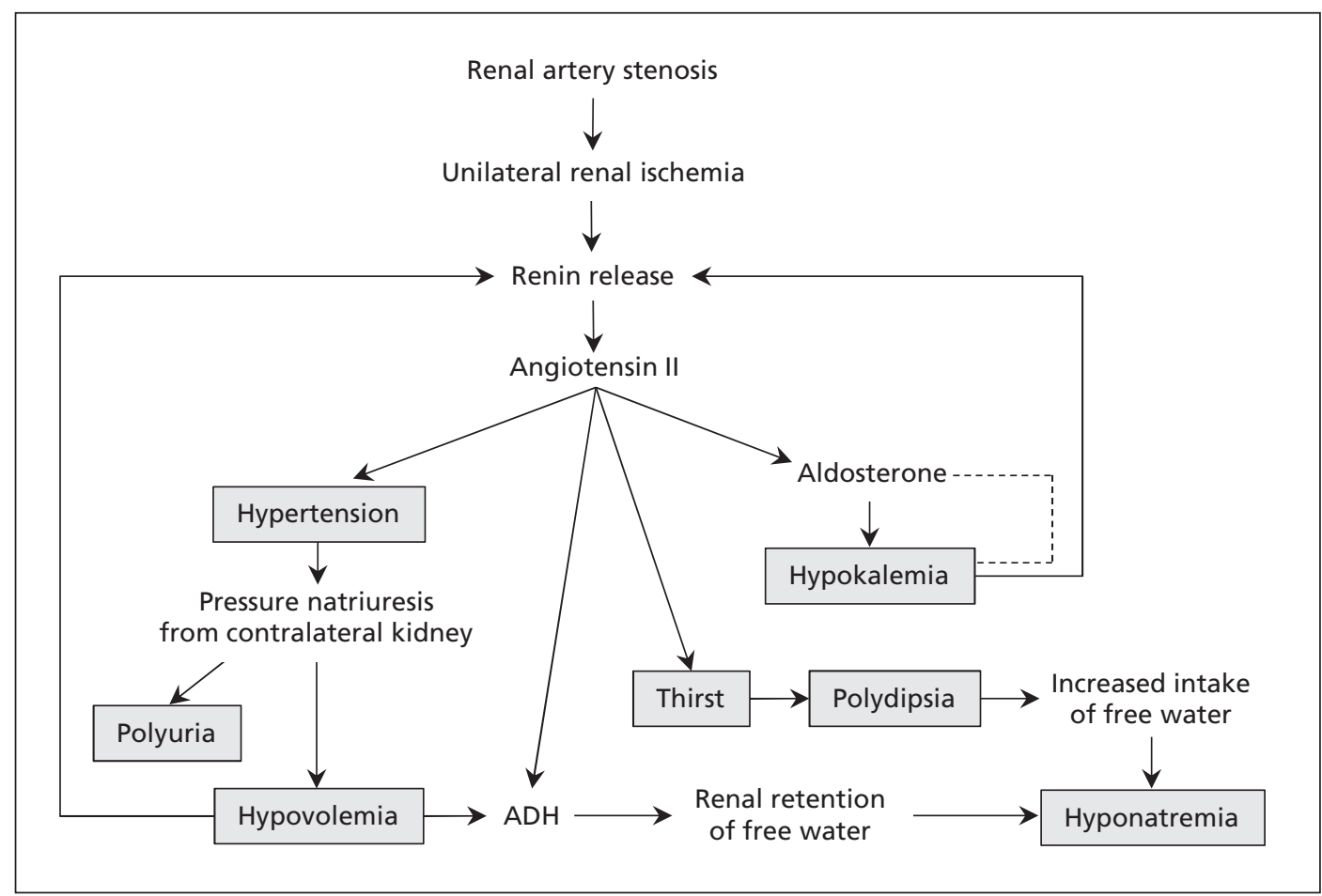

Figure 2: Schematic representation of the pathophysiology of hyponatremic-hypertensive syndrome. Solid lines indicate positive feedback or stimulus; dotted lines indicate negative feedback or inhibition; and boxes indicate clinically apparent signs and symptoms. ADH = antidiuretic hormone. Source: Atkinson et al. ${ }^{3}$ 
begun; however, it was unsuccessful in allowing discontinuation of intravenous fluids without recurrent volume depletion and hyponatremia. Following the addition of an angiotensin-receptor blocker to intensify blockade of the reninangiotensin-aldosterone system, the patient's blood pressure improved and polyuria resolved, and we were able to successfully stop all intravenous fluids and potassium supplementation. Our patient declined surgical intervention, and medical therapy was continued. Following discharge, sodium chloride tablets were gradually reduced and stopped by 6 months. After 1 year of follow-up, our patient has remained well, with no recurrence of hyponatremia, no further hospital admissions and well-controlled blood pressure.

Although combination therapy with an ACE inhibitor and angiotensin-receptor blocker is not recommended for most patients with vascular disease, given the increased risk of adverse events reported in the ONTARGET trial, ${ }^{6}$ our report describes a rare case where dual blockade of the renin-angiotensin-aldosterone system was effective and well tolerated.

\section{References}

1. Wakil A, Ng JM, Atkin SL. Investigating hyponatraemia. BMJ 2011;342:d1118.

2. Agarwal M, Lynn KL, Richards AM, et al. Hyponatremichypertensive syndrome with renal ischemia: an underrecognized disorder. Hypertension 1999;33:1020-4.

3. Kovalski Y, Cleper R, Krause I, et al. Hyponatremic hypertensive syndrome in pediatric patients: Is it really so rare? Pediatr Nephrol 2012;27:1037-40.

4. Atkinson AB, Davies DL, Leckie B, et al. Hyponatraemic hypertensive syndrome with renal-artery occlusion corrected by captopril. Lancet 1979;2:606-9.

5. Robertson GL. Vaptans for the treatment of hyponatremia. Nat Rev Endocrinol 2011;7:151-61.

6. Yusuf S, Teo KK, Pogue J, et al. Telmisartan, ramipril, or both in patients at high risk for vascular events. N Engl J Med 2008;358: 1547-59.

Affiliations: Department of Medicine (Afra, James), University of Calgary, Calgary, Alta.

Contributors: Both authors were responsible for the conception of the manuscript. Kevin Afra drafted the manuscript, and both authors revised it critically and approved the final version submitted for publication.

\section{Call for papers: CMAJ Holiday Reading} CIMAJ Holiday Reading

'Twas months before the holidays and all through CMAJ house not a submission was stirring, making editors grouse. "Holiday Reading time is nigh!" they cried in despair, in hopes that your papers soon would be there.

Submit your evidence-based research on quirky topics, brilliant missives or holidaythemed visuals at http://mc.manuscriptcentral. com/cmaj. Submissions should be no longer than 1200 words. For information contact kelly.clarke@cma.ca

Deadline: October 1, 2013

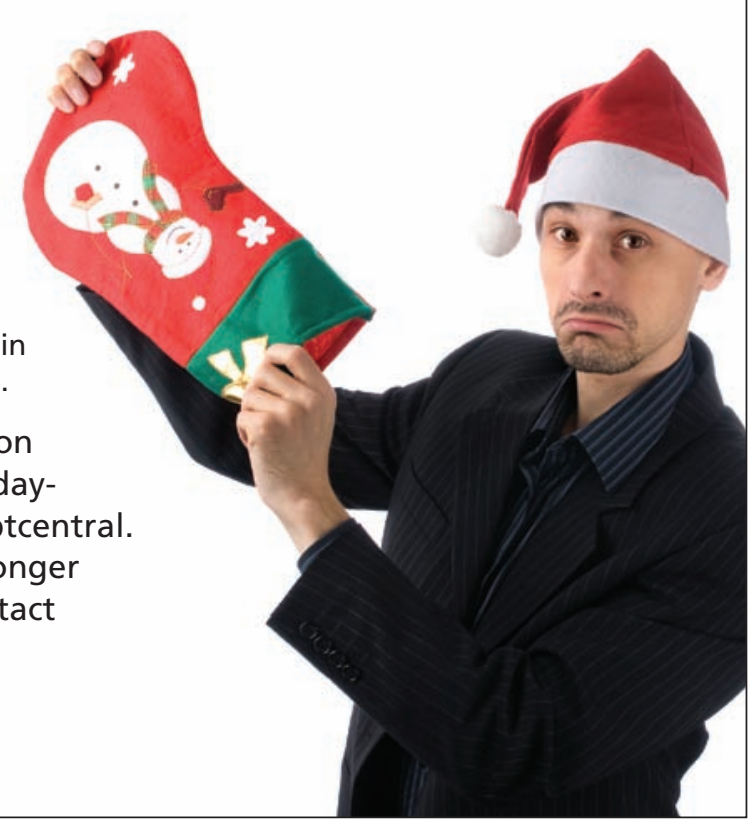

